\title{
What's embodied in a smile?
}

\author{
doi:10.1017/S0140525X10001597
}

Disa A. Sauter and Stephen C. Levinson

Max Planck Institute for Psycholinguistics, 6500AH Nijmegen, The Netherlands.

disa.sauter@mpi.nl stephen.levinson@mpi.nl

http://www.mpi.nl/people/sauter-disa

http://www.mpi.nl/people/levinson-stephen

\begin{abstract}
Differentiation of the forms and functions of different smiles is needed, but they should be based on the empirical data on distinctions that senders and receivers make and on the physical cues that are employed. Such data would allow for a test of whether smiles can be differentiated using perceptual cues alone or whether mimicry or simulation are necessary.
\end{abstract}

The target article proposes an ambitious model of the perception of facial signals, specifically the interpretation of smiling expressions of amusement, dominance, and appeasement. Niedenthal et al. rightly draw attention to the need for differentiation in the forms and functions of different types of smiles, but the distinctions the authors propose lack support from empirical findings, as they themselves concede. This limitation undermines the proposed model. We propose that firm foundations for a model of the perception of emotional signals should be based on the distinctions that senders and receivers make, the cues that are actually employed, and the correspondence between physical cues and subjective experience.

One study has specifically investigated the perception of the three types of smiles that Niedenthal et al. suggest (Hess et al. 2002). When asked to pair facial expressions with vignettes describing dominance, appeasement, and amusement scenarios, Canadians of European extraction associated the amused vignettes with strong Duchenne smiles, but no difference was found between the smiles that were selected for the dominance and appeasement scenarios. For both dominance and appeasement vignettes, responses were distributed across a range of weak- and medium-intensity smiles. These findings demonstrate that amusement is associated with a smile expression, a finding that has recently been replicated cross-culturally and extended to the auditory domain (Sauter, in press; Sauter et al. 2010). However, Hess et al.'s (2002) results suggest that viewers do not reliably differentiate between dominance and appeasement smiles, or, alternatively, that the facial expressions associated with these states are not well established. Niedenthal et al. base their model on the three functionally defined smile 
categories - amused, dominant, and appeasement smiles - but as we have illustrated, it is not established that viewers can differentiate between these three expressions.

There seem, however, to be other distinctions in positive emotion expressions for which there is better empirical grounding. Recently, a growing number of studies have started to distinguish between signals of a range of positive emotions (see Sauter 2010), providing evidence for smile categories other than those suggested by Niedenthal et al. For example, in a production study of posed positive emotion displays, Shiota et al (2003) found that amusement was expressed via open-mouthed smiles, whereas pride was associated with smiles with compressed lips. This finding suggests that displays of some positive affective states are signalled by physically distinct smile configurations, although it did not establish whether viewers are sensitive to these cues. A recent study investigated both the production and perception of spontaneous amused, embarrassed, nervous, and polite smiles, by investigating physical cues and human judgments (Ambadar et al. 2009). Ambadar et al. showed that viewers use variation in morphological and dynamic characteristics of different kinds of smiles. For example, in comparison with smiles perceived to signal politeness, smiles that were perceived by viewers as amused more often included open mouth, larger smile amplitude, larger maximum onset and offset velocity, and longer duration. This demonstrated that viewers' judgments were directly related to the physical cues that differentiated between these expressions. Together, these studies suggest that distinct physical cues are associated with smiles signalling different positive emotions, and that viewers are sensitive to these cues, but the categories are not coincident with those employed in the target article.

Regardless of the specific smile types employed, participants can typically use perceptual, conceptual, and embodiment processes in making these judgments. Additional empirical evidence is necessary for assessing whether an embodiment model actually fits the data. Specifically, Niedenthal et al.'s SIMS model proposes that smiles activate neural regions that cause motor mimicry and somatosensory experience, which form the basis of the viewers' interpretation of the smile they see. Some of the data cited by Niedenthal et al in support of their model are suggestive of emotional mimicry. However, these data are also compatible with the possibility that motor cortex activation in emotion perception tasks reflects downstream associations, rather than playing a primary perceptual role. The proposed model does little to rule out the possibility that viewers may differentiate between different smiles by using perceptual cues alone (perhaps together with conceptual information), before (or in the absence of) any mimicry or simulation.

The authors cite evidence for a causal role of somatosensory cortex in emotion perception from a study showing that transcranial magnetic stimulation (TMS) of this area selectively interferes with performance in a facial expression matching task (Pourtois et al. 2004). However, the manipulation selectively affected the reaction time of judgments of fearful expressions, but did not affect the judgments of happy expressions. Furthermore, no interaction was found with the gaze direction of the faces, and no effect was found on participants' accuracy. The results of the study therefore do not seem to demonstrate a causal role for somatosensory cortex in emotion perception.

In sum, we argue that differentiations of smiles should be based on findings establishing which distinctions senders and receivers make, and what physical cues are actually used by viewers in their judgments. Furthermore, convincing demonstrations that motor cortex activation plays a causal role in emotion perception are lacking, and the possibility remains that viewers can differentiate between smiling expressions from perceptual cues alone, without recourse to simulation. 